\title{
Exogenous regucalcin suppresses the proliferation of human breast cancer MDA-MB-231 bone metastatic cells in vitro
}

\author{
MASAYOSHI YAMAGUCHI $^{1}$ and TOMIYASU MURATA ${ }^{2}$ \\ ${ }^{1}$ Department of Hematology and Medical Oncology, Emory University School of Medicine, Atlanta, GA 30322, USA; \\ ${ }^{2}$ Laboratory of Analytical Neurobiology, Faculty of Pharmacy, Meijo University, Nagoya, Aichi 468-8503, Japan
}

Received January 14, 2015; Accepted September 3, 2015

DOI: $10.3892 / \mathrm{mmr} .2015 .4352$

\begin{abstract}
Regucalcin serves a pivotal role as a suppressor protein in signal transduction in various types of cells and tissues. The regucalcin gene, which is localized on the $\mathrm{X}$ chromosome, consists of seven exons and six introns. Reductions in the gene expression of regucalcin have been suggested to serve a role in hepatocarcinogenesis in animal models and human patients, indicating a potential role as a suppressor protein in cancer. The aim of the current study was to investigate the effect of exogenous regucalcin on cell proliferation in the cloned human breast cancer MDA-MB-231 bone metastatic cell line in vitro. The proliferation of MDA-MB-231 cells was suppressed following the addition of regucalcin (0.1-10 $\mathrm{nM})$ in vitro. The suppression of proliferation was not enhanced in the presence of tumor necrosis factor- $\alpha$, PD98059, staurosporine, Bay K8644, wortmannin, 5,6-dichloro-1- $\beta$-D-ribofuranosylbenzimidazole or gemcitabine. Exogenous regucalcin did not induce cell death in MDA-MB-231 cells in vitro. These data suggest that exogenous regucalcin possesses suppressive effects on the proliferation of human breast cancer MDA-MB-231 bone metastatic cells, and that this effect may be mediated through various intracellular signaling pathways in vitro. Exogenous regucalcin is suggested to function as a suppressor in cancer cell proliferation.
\end{abstract}

\section{Introduction}

Regucalcin is a calcium-binding protein which has been demonstrated to serve a multifunctional role in the regulation of various types of cells and tissues (1-6). The regucalcin gene $(r g n))$, which is located on the $\mathrm{X}$ chromosome, is identified in over 15 species consisting of the regucalcin family, and is highly conserved in vertebrate species throughout

Correspondence to: Dr Masayoshi Yamaguchi, Department of Hematology and Medical Oncology, Emory University School of Medicine, 1365 Clifton Road, Atlanta, GA 30322, USA

E-mail: yamamasa1155@yahoo.co.jp

Key words: regucalcin, breast cancer, MDA-MB-231 cells, cell proliferation, apoptosis, cell signaling, carcinogenesis evolution (7-11). The expression of the regucalcin gene is regulated by various transcription factors including activator protein 1 , nuclear factor I-A1, regucalcin gene promoter region-related protein and $\beta$-catenin, which are modulated through intracellular signaling factors associated with the phosphorylation and dephosphorylation of nuclear proteins in vitro (11). Regucalcin is expressed in the liver, kidney and various other tissues and is regulated by hormonal factors including calcium-regulating hormones, insulin, estrogen and additional steroid hormones $(11,12)$. Regucalcin is translocated from the cytoplasm to the nucleus in various types of cells (13). Regucalcin has been demonstrated to serve a role in the maintenance of intracellular calcium homeostasis, and it inhibits various protein kinases and phosphatases, in addition to inhibiting protein, DNA and RNA syntheses (3-5,13). Additionally, nuclear regucalcin has been demonstrated to regulate the gene expression of various proteins (13). Furthermore, regucalcin suppresses cell proliferation and apoptotic cell death mediated through various signaling factors in normal kidney NRK52E cells and cloned rat hepatoma H4II-E cells in vitro $(14,15)$. Regucalcin has been proposed to serve a physiological role in maintaining cellular homeostasis and function as a regulatory protein of intracellular signaling systems $(5,6)$.

Regucalcin has been demonstrated to possess a pathophysiological role in metabolic disorders (16-18). In addition, regucalcin has been demonstrated to be involved in carcinogenesis (19). The expression of the regucalcin gene and protein have been demonstrated to be reduced in the tumor tissues of animal models and human patients in vivo $(19,20)$. Regucalcin gene expression has been demonstrated to be downregulated in carcinogenesis, suggesting a potential role of regucalcin as a suppressor protein in carcinogenesis (19). Overexpression of endogenous regucalcin has been previously demonstrated to suppress the enhancement of cell proliferation in cloned rat hepatoma H4-II-E cells in vitro (21).

The aim of the current study was to investigate whether exogenous regucalcin possesses a suppressive effect on the proliferation of human cancer cells in vitro.

\section{Materials and methods}

Materials. Dulbecco's modified Eagle's medium (DMEM) with $4.5 \mathrm{~g} / 1$ glucose, L-glutamine and sodium pyruvate and antibiotics (penicillin and streptomycin; $\mathrm{P} / \mathrm{S}$ ) were purchased 
from Invitrogen Life Technologies (Carlsbad, CA, USA). Fetal bovine serum (FBS) was purchased from GE Healthcare Life Sciences (Logan, UT, USA). Gemcitabine was obtained from Hospira, Inc. (Lake Forest, IL, USA), and diluted in Dulbecco's modified phosphate-buffered saline (PBS). Tumor necrosis factor- $\alpha$ (TNF- $\alpha$ ) was purchased from R\&D Systems, Inc. (Minneapolis, MN, USA).PD98059, staurosporine, Bay K8644, wortmannin, 5,6-dichloro-1- $\beta$-D-ribofuranosylbenzimidazole (DRB) and all additional reagents were purchased from Sigma-Aldrich (St. Louis, MO, USA) unless otherwise specified.

Regucalcin. Regucalcin was isolated from rat liver cytosol as described previously (1). The livers were perfused with Tris- $\mathrm{HCl}$ buffer (pH 7.4), containing $100 \mathrm{mM}$ Tris, $120 \mathrm{mM}$ $\mathrm{NaCl}$ and $4 \mathrm{mM} \mathrm{KCl}$, and cooled to $4^{\circ} \mathrm{C}$. The livers were subsequently removed, cut into small pieces, suspended 1:4 (w/v) in Tris-HCl buffer ( $\mathrm{pH} \mathrm{7.4),} \mathrm{and} \mathrm{homogenized} \mathrm{in}$ a Potter-Elvehjem homogenizer (Takashima System Ltd., Tokyo, Japan) with a Teflon pestle (1). The homogenate was spun at 5,500 $\mathrm{x} \mathrm{g}$ in a refrigerated centrifuge for $10 \mathrm{~min}$, and the supernatant was spun at $105,000 \mathrm{x}$ g for $60 \mathrm{~min}$ at $4^{\circ} \mathrm{C}$. The resulting supernatant was purified to electrophoretic homogeneity by gel filtration using Sephadex G-75 and G-50, followed by ion-exchange chromatography on diethylaminoethyl-cellulose (4). The purity of the isolated regucalcin was analyzed using gel electrophoresis and western blot analysis, which confirmed that it did not contain other proteins.

Breast cancer MDA-MB-231-bone metastatic cells. Breast cancer MDA-MB-231 bone metastatic cells lack estrogen, progesterone and human epidermal growth factor type 2 receptors, and are therefore considered as triple negative (22). However, the cells express epidermal growth factor, transforming growth factor- $\alpha$ and Wnt7B oncogene, and activation of these receptors and its downstream signaling events enhances the migration, proliferation, invasion and progression of the malignant phenotype of these cells. The MDA-MB-231 bone metastatic cells were provided by Dr Toshi Yoneda (The University of Texas, San Antonio, TX, USA) (22).

Cell proliferation in MDA-MB-231 cells. Breast cancer MDA-MB-231 cells $\left(1 \times 10^{5} / \mathrm{ml}\right.$ per well) were cultured in a 24-well plate in DMEM containing $10 \% \mathrm{FBS}$ and $1 \% \mathrm{P} / \mathrm{S}$ in the presence or absence of regucalcin $(0.01,0.1,05,1$ or $10 \mathrm{nM})$ for 1, 2, 3 and 7 days. In separate experiments, MDA-MB-231 cells $\left(1 \times 10^{5} / \mathrm{ml}\right.$ per well) were cultured in DMEM containing $10 \% \mathrm{FBS}$ and $1 \% \mathrm{P} / \mathrm{S}$ in the presence of TNF- $\alpha(1 \mathrm{ng} / \mathrm{ml})$, Bay K8644 $(1 \mu \mathrm{M})$, PD98059 $(1 \mu \mathrm{M})$, staurosporine $(0.1 \mu \mathrm{M})$, wortmannin $(1 \mu \mathrm{M})$ or DRB $(1 \mu \mathrm{M})$ for 3 days. Following culture, the number of cells was counted.

Cell death in MDA-MB-231 cells. Breast cancer MDA-MB-231 cells $\left(1 \times 10^{5} / \mathrm{ml}\right.$ per well) were cultured in a $24-w e l l$ plate in DMEM containing 10\% FBS and 1\% P/S in the absence of regucalcin for 7 days, until cells were confluent. Subsequently, the cells were cultured in the presence or absence of regucalcin $(0.1,1$ or $10 \mathrm{nM})$ with or without gemcitabine $(10,50,100,250$, and $1,000 \mathrm{nM}$ ) for 7 days. Following culture, the number of cells was counted.
Cell counting. Following trypsinization of each culture dish in $0.2 \%$ trypsin with $0.02 \%$ EDTA in $\mathrm{Ca}^{2+} / \mathrm{Mg}^{2+}$-free PBS for 2 min at $37^{\circ} \mathrm{C}$, the detached cells were collected by centrifugation at $12 \mathrm{x} \mathrm{g}$ for $5 \mathrm{~min}$ at $4^{\circ} \mathrm{C}$ (Eppendorf $5810 \mathrm{R}$ ). The cells were resuspended in PBS solution and stained with eosin. Cell numbers were counted under a microscope (Olympus MTV-3; Olympus Corporation, Tokyo, Japan) using a hemocytometer (Brightline; Sigma-Aldrich). For each dish, the cells were counted twice from which the average was calculated. Cell numbers are presented as the number/well of plate.

Statistical analysis. Data are presented as the mean \pm standard deviation. Statistical significance was determined using GraphPad InStat software, version 3 (GraphPad Software, Inc., La Jolla, CA, USA). Multiple comparisons were conducted using a one-way analysis of variance and a Tukey-Kramer multiple comparisons post-test for parametric data. $\mathrm{P}<0.05$ was considered to indicate a statistically significant difference.

\section{Results}

The effect of exogenous regucalcin on the proliferation of breast cancer MDA-MB-231 bone metastatic cells in vitro is presented in Fig. 1. MDA-MB-231 cells were cultured in the presence of exogenous regucalcin (0.1-10 nM) for 1-7 days. The number of cells increased with the increasing duration of culture. The addition of exogenous regucalcin reduced the increase in cell number, indicating that cell proliferation was suppressed by the physiological concentrations of serum regucalcin (23).

The suppressive effects of regucalcin $(1 \mathrm{nM})$ on cell proliferation in MDA-MB-231 cells were not enhanced in the presence of TNF- $\alpha$ (1 ng/ml), an enhancer of nuclear factor- $\kappa B$ (NF- $\kappa$ B) signaling (24) or Bay K8644 $(1 \mu \mathrm{M})$, an agonist of $\mathrm{Ca}^{2+}$ influx in cells (25), which resulted in significantly reduced cell numbers when applied alone (Fig. 2). In addition, the effects of exogenous regucalcin in reducing cell proliferation were not enhanced in the presence of PD98059 $(1 \mu \mathrm{M})$, a mitogen-activated protein kinase (MAPK) inhibitor (26) or staurosporine $(0.1 \mu \mathrm{M})$, an inhibitor of protein kinase $\mathrm{C}(27)$, which caused a significant reduction in cell numbers (Fig. 3). Furthermore, the suppressive effects of regucalcin on cell proliferation were not enhanced in the presence of wortmannin $(1 \mu \mathrm{M})$, an inhibitor of phosphatidylinositol 3-kinase (PI3K) (28) or DRB (1 $\mu \mathrm{M})$, an inhibitor of transcriptional activity via RNA polymerase II inhibition (29) (Fig. 4).

The suppressive effects of regucalcin on the proliferation of MDA-MB-231 cells were investigated in the presence of gemcitabine, an antitumor agent, which induces nuclear DNA damage (30). The addition of gemcitabine (50-500 $\mathrm{nM})$ to MDA-MB-231 cultures reduced cell proliferation (Fig. 5A). This effect was not altered by the application of regucalcin $(1 \mathrm{nM})$ with gemcitabine (Fig. 5B). The addition of regucalcin $(1 \mathrm{nM})$ significantly reduced cell numbers in the presence of gemcitabine $(10 \mathrm{nM})$.

The effect of regucalcin on cell death in breast cancer MDA-MB-231 cells is presented in Fig. 6. Cells were cultured for 7 days until confluent, and subsequently cultured for 7 days in the presence of regucalcin $(0.1$ or $1 \mathrm{nM})$ with or without gemcitabine $(100 \mathrm{nM})$. The addition of regucalcin was not 
A

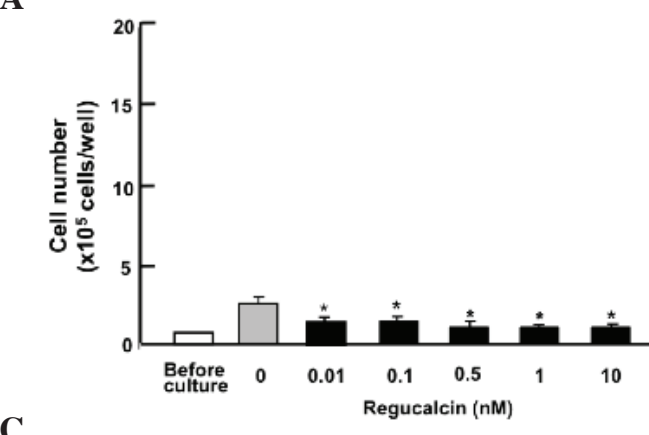

C

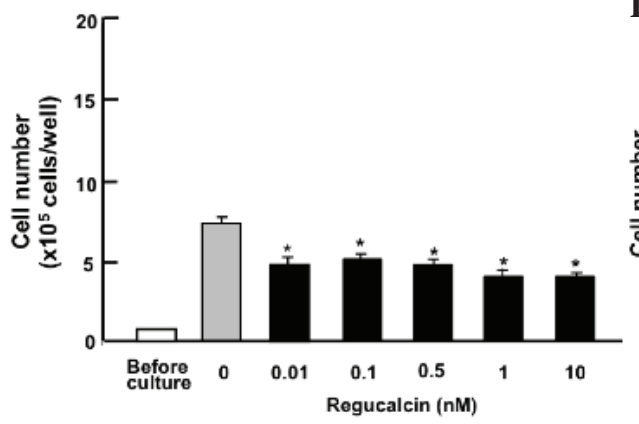

B

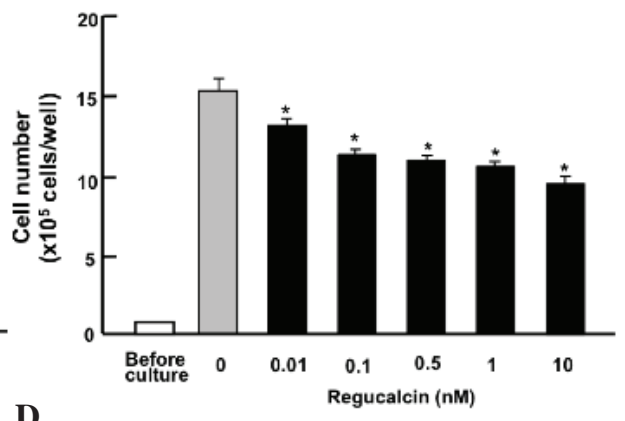

D

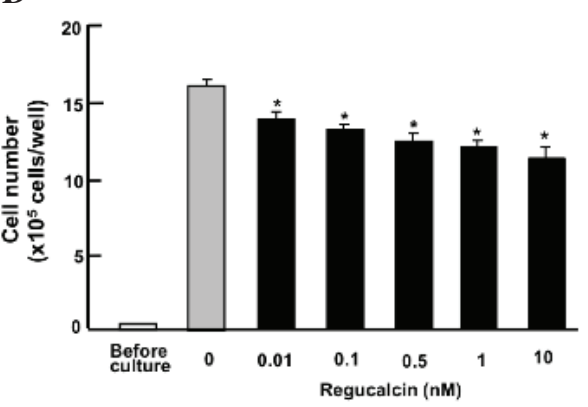

Figure 1. Exogenous regucalcin suppresses the proliferation of MDA-MB-231 cells in vitro. Cells were cultured in the presence or absence of regucalcin (0.1-10 nM) for (A) 1, (B) 2, (C) 3 or (D) 7 days. Following culture, the number of cells were counted. Data are presented as the mean \pm standard deviation of 2 replicate wells per data set using different dishes and cell preparations. "P<0.001 vs. control (grey bar). One way analysis of variance and the Tukey-Kramer post-test were used.

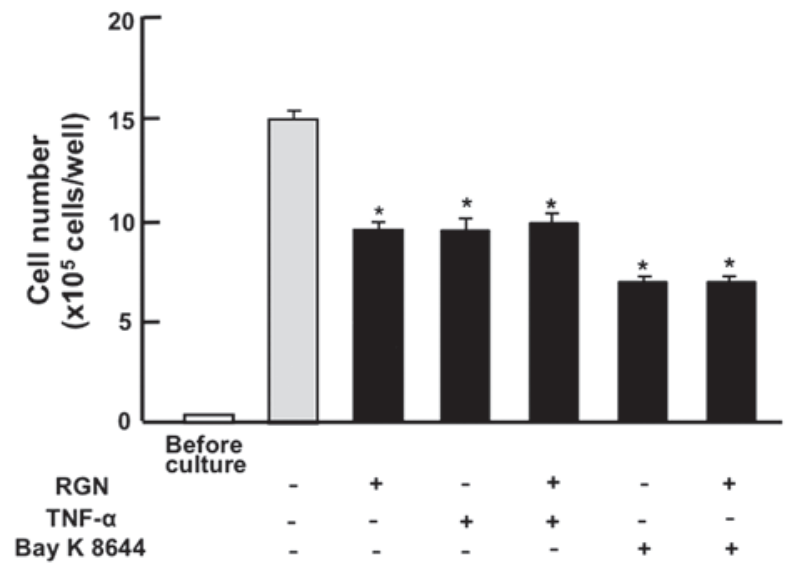

Figure 2. Suppressive effects of exogenous RGN on the proliferation of MDA-MB-231 cells in the presence of TNF- $\alpha$ or Bay K8644 in vitro. Cells were cultured for 3 days in the presence of RGN (1 nM) with or without TNF- $\alpha(1 \mathrm{ng} / \mathrm{ml})$ or Bay K8644 $(1 \mu \mathrm{M})$. Following culture, the number of cells were counted. Data are presented as the mean \pm standard deviation of 2 replicate wells per data set using different dishes and cell preparations. ${ }^{*} \mathrm{P}<0.001$ vs. control (grey bar). One way analysis of variance and the Tukey-Kramer post-test were used. RGN, regucalcin; TNF- $\alpha$, tumor necrosis factor- $\alpha$.

observed to significantly affect the cell number, however culture with gemcitabine reduced cell number. Therefore, it is suggested that regucalcin does not induce cell death.

\section{Discussion}

Regucalcin has been demonstrated to serve a multifunctional role in the regulation of cell function by suppressing various

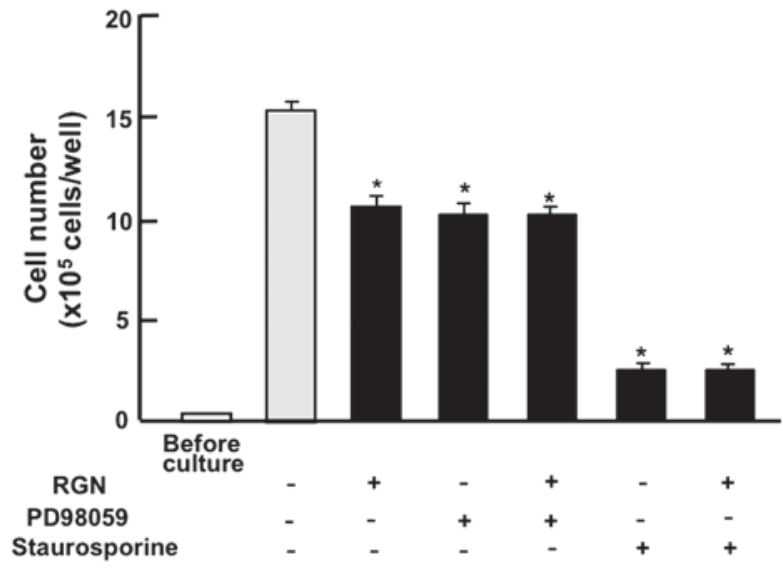

Figure 3. Suppressive effects of exogenous RGN on the proliferation of MDA-MB-231 cells in the presence of PD98059 or staurosporine in vitro. Cells were cultured for 3 days in the presence of RGN ( $1 \mathrm{nM}$ ) with or without PD98059 $(1 \mu \mathrm{M})$ or staurosporine $(0.1 \mu \mathrm{M})$. Following culture, the number of cells were counted. Data are presented as the mean \pm standard deviation of 2 replicate wells per data set using different dishes and cell preparations. ${ }^{*} \mathrm{P}<0.001$ vs. control (grey bar). One way analysis of variance and the Tukey-Kramer post-test were used. RGN, regucalcin.

signaling pathways in various types of cells and tissues (4-6). Previous studies have demonstrated that regucalcin serves a potential role as a suppressor of cell proliferation and carcinogenesis $(14,18,19)$. Regucalcin gene expression was observed to be downregulated in the tumor tissues of human patients (20) and in human cancer cells $(18,31)$. The current study demonstrated that exogenous regucalcin possesses suppressive effects on the proliferation of human breast cancer 


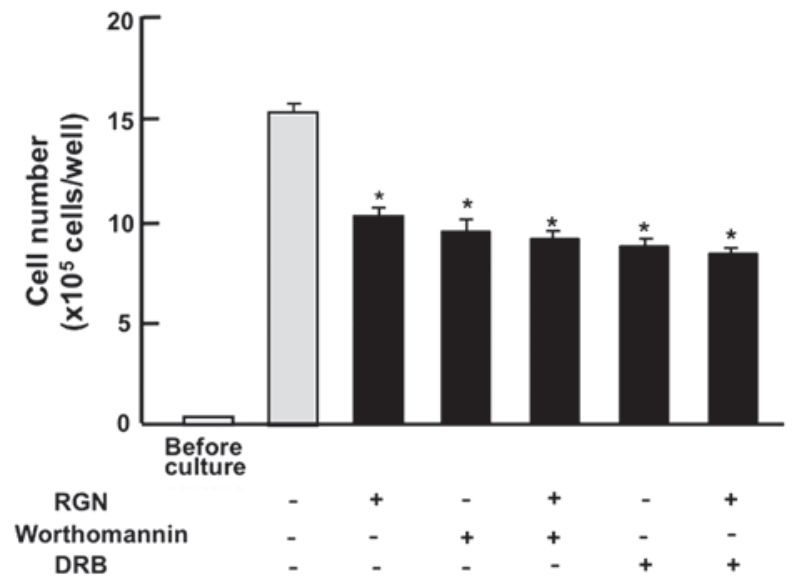

Figure 4. Suppressive effects of exogenous RGN on the proliferation of MDA-MB-231 cells in the presence of wortmannin or DRB in vitro. Cells were cultured for 3 days in the presence of RGN $(1 \mathrm{nM})$ with or without wortmannin $(1 \mu \mathrm{M})$ or DRB $(1 \mu \mathrm{M})$. Following culture, the number of cells were counted. Data are presented as the mean \pm standard deviation of 2 replicate wells per data set using different dishes and cell preparations. $\mathrm{P}<0.001$ vs. control (grey bar). One way analysis of variance and the Tukey-Kramer post-test were used. RGN, regucalcin; DRB, 5,6-dichloro-1- $\beta$-D-ribofurano sylbenzimidazole.

A
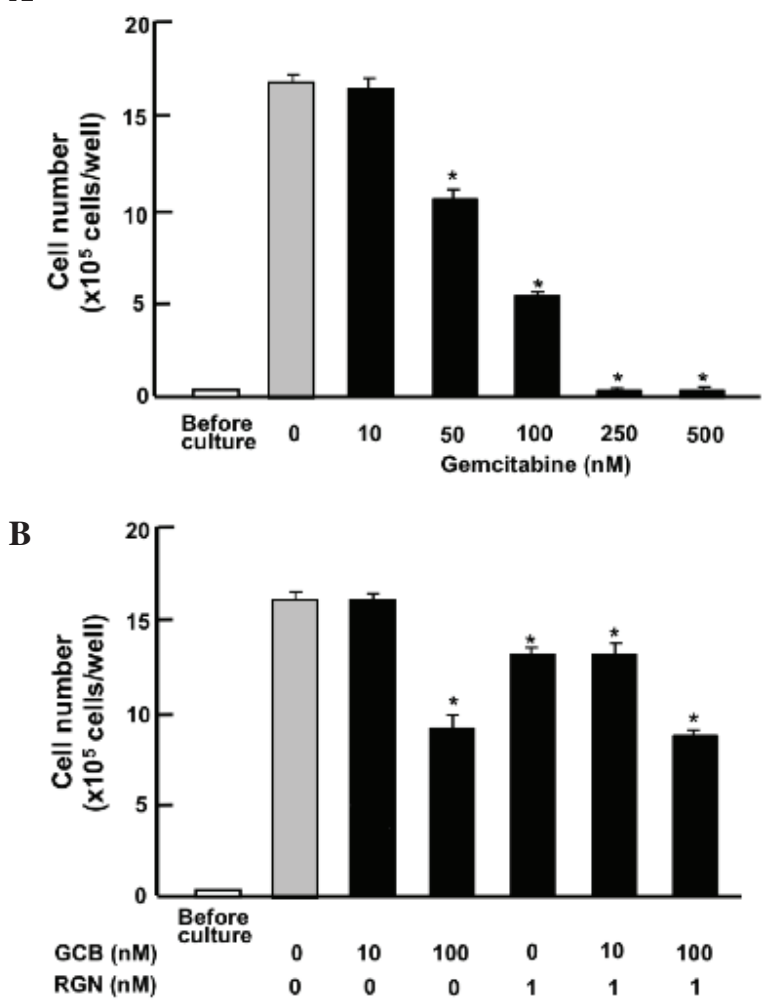

Figure 5. Suppressive effects of exogenous RGN on the proliferation of MDA-MB-231 cells in the presence of GCB in vitro. (A) Cells were cultured for 7 days in the presence of GCB (10-500 nM). (B) Cells were cultured for 7 days in the presence of RGN (1 nM) with or without GCB $(10$ or $100 \mathrm{nM})$. Following culture, the number of cells were counted. Data are presented as the mean \pm standard deviation of 2 replicate wells per data set using different dishes and cell preparations. ${ }^{*} \mathrm{P}<0.001$ vs. control (white bar). One way analysis of variance and the Tukey-Kramer post-test were used. RGN, regucalcin; GCB, gemcitabine.

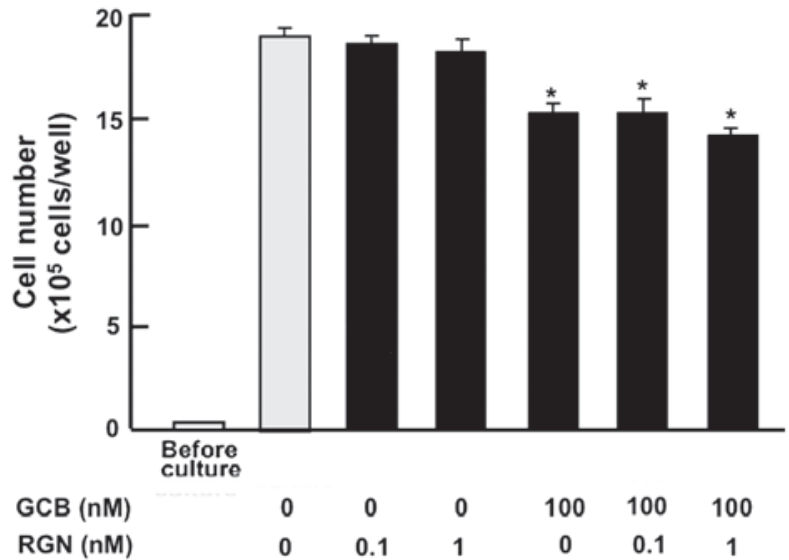

Figure 6. Effect of exogenous RGN on cell death in MDA-MB-231 cells in the presence of GCB in vitro. Cells were cultured for 7 days until confluent, and subsequently cultured for 7 days in the presence of RGN (0.1 or $1 \mathrm{nM})$ with or without GCB $(100 \mathrm{nM})$. Following culture, the number of cells were counted. Data are presented as the mean \pm standard deviation of 2 replicate wells per data set using different dishes and cell preparations. ${ }^{*} \mathrm{P}<0.001$ vs. control (grey bar). One way analysis of variance and the Tukey-Kramer post-test were used. RGN, regucalcin; GCB, gemcitabine.

MDA-MB-231 bone metastatic cells in vitro, however was not observed to affect cell death.

Overexpression of endogenous regucalcin has been demonstrated to suppress the proliferation of cloned rat hepatoma H4-II-E cells in vitro $(14,18,21)$. Regucalcin has been demonstrated to result in $G_{1}$ and $G_{2} / M$ phase cell cycle arrest in H4-II-E cells (32). In addition, overexpression of endogenous regucalcin has been demonstrated to have suppressive effects on cell proliferation inducing $\mathrm{G}_{1}$ and $\mathrm{G}_{2} / \mathrm{M}$ phase cell cycle arrest in cloned normal rat kidney proximal tubular epithelial NRK52E cells in vitro (33). The suppressive effects of endogenous regucalcin on cell proliferation may be mediated through the inhibition of various $\mathrm{Ca}^{2+}$ signaling-dependent protein kinases, protein phosphatases and PI3K activities and the suppression of c-Myc, H-Ras, c-Jun and chk2 mRNA expression, or the enhancement of p53 and Rb mRNA expression $(14,19,34,35)$. Furthermore, regucalcin has been demonstrated to suppress cytoplasmic protein synthesis and nuclear DNA and RNA synthesis $(13,14)$.

The current study demonstrated that the suppressive effects of exogenous regucalcin on the proliferation of MDA-MB-231 cells were not modulated in the presence of various inhibitors that regulate intracellular signaling pathways in vitro. The suppressive effects of regucalcin on the proliferation of MDA-MB-231 cells were not enhanced in the presence of: TNF- $\alpha$, an enhancer of NF- $\kappa \mathrm{B}$ signaling (24); Bay K8644, an agonist of $\mathrm{Ca}^{2+}$ entry in cells (25); PD98059, an MAPK inhibitor (26); staurosporine, an inhibitor of calcium-dependent protein kinase C (27); or wortmannin, an inhibitor of PI3K (28). These data suggest that exogenous regucalcin stimulates various intracellular signaling pathways to suppress cell proliferation in human breast cancer MDA-MB-231 cells. Regucalcin has been demonstrated to bind the plasma membranes of rat liver cells in vitro (36). Therefore, it may be possible that exogenous regucalcin binds to the plasma membranes of breast cancer MDA-MB-231 cells, 
and potentially regulates intracellular signaling pathways that suppress cell proliferation. In addition, the suppressive effects of regucalcin on cell proliferation were not enhanced in the presence of DRB, an inhibitor of transcriptional activity via RNA polymerase II inhibition (29). The intracellular signals of exogenous regucalcin may be transmitted into the nucleus to suppress transcriptional regulation in human breast cancer MDA-MB-231 cells.

Overexpression of endogenous regucalcin has been demonstrated to have suppressive effects on apoptotic cell death in rat hepatoma H4-II-E cells and normal rat kidney NRK52-E cells, which were increased through various signaling pathways in the cytoplasm and nucleus in vitro (15). In the current study, exogenous regucalcin did not induce cell death in human breast cancer MDA-MB-231 cells in vitro, indicating that regucalcin does not stimulate cell death. This effect was not enhanced in the presence of gemcitabine, an antitumor agent, which induces nuclear DNA damage (30). This observation may support the theory that the intracellular signaling by exogenous regucalcin may be transmitted to regulate nuclear function in breast cancer cells.

In conclusion, the current study demonstrated that exogenous regucalcin possesses suppressive effects on the proliferation of human breast cancer MDA-MB-231 bone metastatic cells in vitro. This suggests that exogenous regucalcin serves a role as a suppressor of the proliferation of human cancer cells.

\section{References}

1. Yamaguchi $\mathrm{M}$ and Yamamoto T: Purification of calcium binding substance from soluble fraction of normal rat liver. Chem Pharm Bull (Tokyo) 26: 1915-1918, 1978.

2. Yamaguchi M and Sakurai T: Inhibitory effect of calcium-binding protein regucalcin on $\mathrm{Ca}^{2(+)}$-activated DNA fragmentation in rat liver nuclei. FEBS Lett 279: 281-284, 1991.

3. Shimokawa N and Yamaguchi M: Molecular cloning and sequencing of the cDNA coding for a calcium-binding protein regucalcin from rat liver. FEBS Lett 327: 251-255, 1993.

4. Yamaguchi M: Role of regucalcin in calcium signaling. Life Sci 66: 1769-1780, 2000.

5. Yamaguchi M: Role of regucalcin in maintaining cell homeostasis and function (review). Int J Mol Med 15: 371-389, 2005.

6. Yamaguchi M: Regucalcin and cell regulation: Role as a suppressor protein in signal transduction. Mol Cell Biochem 353 101-137, 2011.

7. Shimokawa N, Matsuda Y and Yamaguchi M: Genomic cloning and chromosomal assignment of rat regucalcin gene. Mol Cell Biochem 151: 157-163, 1995.

8. Thiselton DL, McDowall J, Brandau O, Ramser J, d'Esposito F, Bhattacharya SS, Ross MT, Hardcastle AJ and Meindl A: An integrated, functionally annotated gene map of the DXS8026-ELK1 interval on human Xp11.3-Xp11.23: Potential hotspot for neurogenetic disorders. Genomics 79: 560-572, 2002.

9. Yamaguchi M, Makino R and Shimokawa N: The 5' end sequences and exon organization in rat regucalcin gene. Mol Cell Biochem 165: 145-150, 1996.

10. Misawa $\mathrm{H}$ and Yamaguchi $\mathrm{M}$ : The gene of $\mathrm{Ca}^{2+}$-binding protein regucalcin is highly conserved in vertebrate species. Int $\mathrm{J}$ Mol Med 6: 191-196, 2000.

11. Yamaguchi M: The transcriptional regulation of regucalcin gene expression. Mol Cell Biochem 346: 147-171, 2011.

12. Yamaguchi M: Hormonal Regulation of regucalcin gene expression: Involvement in cell metabolism. Horm Stud 1: 1, 2013

13. Yamaguchi M: Role of regucalcin in cell nuclear regulation: Involvement as a transcription factor. Cell Tissue Res 354: 331-341, 2013.
14. Yamaguchi M: Suppressive role of regucalcin in liver cell proliferation: Involvement in carcinogenesis. Cell Prolif 46: 243-253, 2013.

15. Yamaguchi M: The anti-apoptotic effect of regucalcin is mediated through multisignaling pathways. Apoptosis 18: 1145-1153, 2013.

16. Yamaguchi M: Regucalcin and metabolic disorder: Osteoporosis and hyperlipidemia are induced in regucalcin transgenic rats. Mol Cell Biochem 341: 119-133, 2010.

17. Yamaguchi $\mathrm{M}$ and Murata $\mathrm{T}$ : Involvement of regucalcin in lipid metabolism and diabetes. Metabolism 62: 1045-1051, 2013.

18. Yamaguchi M: Regucalcin as a potential biomarker for metabolic and neuronal diseases. Mol Cell Biochem 391:157-166, 2014.

19. Yamaguchi M: Involvement of regucalcin as a suppressor protein in human carcinogenesis: Insight into the gene therapy. J Cancer Res Clin Oncol 141: 1333-1341, 2015.

20. Murata T and Yamaguchi M: Alternatively spliced variants of the regucalcin gene in various human normal and tumor tissues. Int J Mol Med 34: 1141-1146, 2014.

21. Misawa H, Inagaki S and Yamaguchi M: Suppression of cell proliferation and deoxyribonucleic acid synthesis in the cloned rat hepatoma H4-II-E cells overexpressing regucalcin. J Cell Biochem 84: 143-149, 2001

22. Hiraga T, Williams PJ, Mundy GR and Yoneda T: The bisphosphonate ibandronate promotes apoptosis in MDA-MB-231 human breast cancer cells in bone metastases. Cancer Res 61: 4418-4424, 2001.

23. Yamaguchi $M$ and Isogai $M$ : Tissue concentration of calcium-binding protein regucalcin in rats by enzyme-linked immunoadsorbent assay. Mol Cell Biochem 122: 65-68, 1993.

24. Lee ZH, Kwack K, Kim KK, Lee SH and Kim HH: Activation of c-Jun N-terminal kinase and activator protein 1 by receptor activator of nuclear factor kappaB. Mol Pharmacol 58: 1536-1545, 2000.

25. Cano-Abad MF, Villarroya M, García AG, Gabilan NH and López MG: Calcium entry through L-type calcium channels causes mitochondrial disruption and chromaffin cell death. J Biol Chem 276: 39695-39704, 2001.

26. Chen S, Wang Y, Ruan W, Wang X and Pan C: Reversing multidrug resistance in hepatocellular carcinoma cells by inhibiting extracellular signal-regulated kinase/mitogen-activated protein kinase signaling pathway activity. Oncol Lett 8: 2333-2339, 2014.

27. Chen QW, Edvinsson L and Xu CB: Role of ERK/MAPK in endothelin receptor signaling in human aortic smooth muscle cells. BMC Cell Biol 10: 52, 2009.

28. Serrano-Nascimento C, da Silva Teixeira S, Nicola JP, Nachbar RT, Masini-Repiso AM and Nunes MT: The acute inhibitory effect of iodide excess on sodium/iodide symporter expression and activity involves the PI3K/Akt signaling pathway. Endocrinology 155: 1145-1156, 2014.

29. Palangat M, Grass JA, Langelier MF, Coulombe B and Landick R: The RPB2 flap loop of human RNA polymerase II is dispensable for transcription initiation and elongation. Mol Cell Biol 31: 3312-3325, 2011.

30. Tang SC and Chen YC: Novel therapeutic targets for pancreatic cancer. World J Gastroenterol 20: 10825-10844, 2014.

31. Maia C, Santos C, Schmitt F and Socorro S: Regucalcin is under-expressed in human breast and prostate cancers: Effect of sex steroid hormones. J Cell Biochem 107: 667-676, 2009.

32. Yamaguchi $M$ and Daimon Y: Overexpression of regucalcin suppresses cell proliferation in cloned rat hepatoma H4-II-E cells: Involvement of intracellular signaling factors and cell cycle-related genes. J Cell Biochem 95: 1169-1177, 2005.

33. Nakagawa T, Sawada N and Yamaguchi M: Overexpression of regucalcin suppresses cell proliferation of cloned normal rat kidney proximal tubular epithelial NRK52E cells. Int J Mol Med 16: 637-643, 2005.

34. Tsurusaki Y and Yamaguchi M: Overexpression of regucalcin modulates tumor-related gene expression in cloned rat hepatoma H4-II-E cells. J Cell Biochem 90: 619-626, 2003.

35. Tsurusaki $\mathrm{Y}$ and Yamaguchi M: Role of regucalcin in liver nuclear function: Binding of regucalcin to nuclear protein or DNA and modulation of tumor-related gene expression. Int J Mol Med 14: 277-281, 2004

36. Yamaguchi M, Mori S and Kato S: Calcium-binding protein regucalcin is an activator of $\left(\mathrm{Ca}^{2+}-\mathrm{Mg}^{2+}\right)$-adenosine triphosphatase in the plasma membranes of rat liver. Chem Pharm Bull (Tokyo) 36: 3532-3539, 1988 